Supporting Information

\title{
Role of Cu-Mg-Al mixed oxide catalysts in lignin depolymerization in supercritical ethanol
}

Xiaoming Huang, ${ }^{a}$ Ceylanpinar Atay, ${ }^{b}$ Tamás I. Korányi, ${ }^{a}$ Michael D. Boot, ${ }^{c}$ and Emiel J.M. Hensen $^{a, *}$

${ }^{a}$ Schuit Institute of Catalysis, Inorganic Materials Chemistry, Eindhoven University of Technology, P.O. Box 513, 5600 MB Eindhoven (The Netherlands),

${ }^{b}$ Chemical Engineering Department, Istanbul Technical University, 34469, Maslak, Istanbul, Turkey

${ }^{c}$ Combustion Technology, Department of Mechanical Engineering, Eindhoven University of Technology, P.O. Box 513, 5600 MB Eindhoven (The Netherlands)

*Correspondence to: e.j.m.hensen@tue.nl

Table of Contents

1. Supplemental Results

1.1. Ethanol conversion product (with lignin).

1.2. Ethanol conversion product list (without lignin)

1.3. Result of blank reaction (without catalyst)

1.4. Identification of peaks in HSQC NMR

1.5. XPS analysis of the catalysts.

1.6. GPC analysis of the lignin and its residues.

1.7. Gas phase products analysis.

1.1 Ethanol conversion product (with lignin).

Table S1. Ethanol product list obtained from the catalytic reaction of lignin at $340{ }^{\circ} \mathrm{C}$ for $4 \mathrm{~h}$ over the $\mathrm{Cu}_{20} \mathrm{MgAl}_{(4)}$ catalyst in ethanol (Table 3 entry 4). 


\begin{tabular}{|c|c|c|c|c|}
\hline $\begin{array}{l}\text { Retention } \\
\text { time (min) }\end{array}$ & Area & Compound Name & $\begin{array}{l}\text { Amount of } \\
\text { products }(\mathrm{mg})\end{array}$ & $\begin{array}{l}\text { Weight RF to C12 } \\
\text { Alkane }\end{array}$ \\
\hline 3.334 & 95492.9 & E-Alkane-C2-30: Ethane & 14.31 & 0.946 \\
\hline 3.374 & 451629.4 & E-Alkane-C3-42: Propene & 63.31 & 1.012 \\
\hline 3.471 & 386970.8 & E-Alkene-C4-56:2-Butene, & 54.30 & 1.011 \\
\hline 3.504 & 496654.4 & E-Alkene-C4-56:2-Butene, & 69.69 & 1.011 \\
\hline 3.535 & 389759.5 & E-Alkene-C4-56:2-Butene, & 54.69 & 1.011 \\
\hline 3.707 & 755228.6 & E-Alkene-C5-70: 1-Pentene & 105.78 & 1.013 \\
\hline 3.775 & 143185.5 & E-Alkene-C5-70: 2-Methyl-1-butene & 20.06 & 1.013 \\
\hline 4.423 & 156218.9 & E- Alkene-C6-84: 2-Hexene & 29.55 & 1.012 \\
\hline 4.555 & 26847 & E-Alkene-C6-84: 2-Hexene & 3.77 & 1.011 \\
\hline 4.62 & 32182.7 & E-Alkene-C6-84: 2-Pentene, 2-methyl- & 4.51 & 1.012 \\
\hline 4.511 & 22251.2 & E-Alkene-C6-84: 2-Pentene, 3-methyl- & 3.12 & 1.012 \\
\hline 5.778 & 37327.8 & E-Alkene-C7-98: 3-Heptene & 5.24 & 1.011 \\
\hline 5.903 & 20448.1 & E-Alkene-C7-98: 2-Heptene & 2.87 & 1.011 \\
\hline 15.37 & 83695.3 & E-Alkane-C9-128: Heptane, 3,4-dimethyl- & 11.92 & 0.996 \\
\hline 5.232 & 18101.1 & E-Alcohol-C3-60: Propanol & 4.53 & 0.567 \\
\hline 7.216 & 153895.5 & E-Alcohol-C4-70: 1,3-Butadien-1-ol & 31.76 & 0.687 \\
\hline 7.884 & 2742610 & E-Alcohol-C4-72: 2-Buten-1-ol & 582.22 & 0.668 \\
\hline 8.486 & 530788 & E-Alcohol-C4-72: 2-Buten-1-ol & 112.68 & 0.668 \\
\hline 7.664 & 10235039 & E-Alcohol-C4-74: 1-Butanol & 2233.13 & 0.650 \\
\hline 8.341 & 45473.3 & E-Alcohol-C5-88: 2-Pentanol & 9.10 & 0.709 \\
\hline 10.073 & 53317.6 & E-Alcohol-C5-88: 1-Butanol, 2-methyl- & 10.67 & 0.709 \\
\hline 14.752 & 188280 & E-Alcohol-C6-100: 2-Penten-1-ol, 2-methyl- & 34.91 & 0.765 \\
\hline 14.966 & 147454.5 & E-Alcohol-C6-100: 2-Penten-1-ol, 2-methyl- & 27.34 & 0.765 \\
\hline 14.553 & 57474.5 & E-Alcohol-C6-100: 3-Hexen-1-ol & 10.66 & 0.765 \\
\hline 13.356 & 39281.6 & E-Alcohol-C6-100: 4-Hexen-1-ol & 7.28 & 0.765 \\
\hline 15.059 & 560209.1 & E-Alcohol-C6-102: 1-Hexanol & 105.95 & 0.750 \\
\hline 14.256 & 36745.3 & E-Alcohol-C6-102: 1-Pentanol, 3-methyl- & 6.95 & 0.750 \\
\hline 13.842 & 44119.1 & E-Alcohol-C6-102: 1-Butanol, 2-ethyl- & 8.34 & 0.750 \\
\hline 13.263 & 21019 & E-Alcohol-C7-116: 2-Hexanol, 3-methyl- & 3.82 & 0.781 \\
\hline 3.607 & 939086.3 & E-Aldehyde-C2-44-Acetaldehyde & 415.39 & 0.321 \\
\hline 5.255 & 224537.6 & E-Aldehyde-C4-72: Butanal & 53.96 & 0.590 \\
\hline 12.443 & 64317.6 & E-Aldehyde-C6-98 : 2-Butenal, 2-ethyl- & 12.61 & 0.723 \\
\hline 5.316 & 1352195 & E-Ester-C4-88: Ethyl acetate & 594.54 & 0.323 \\
\hline 11.214 & 63335.2 & E-Ester-C6-116: Acetic acid, butyl ester & 18.41 & 0.488 \\
\hline 10.426 & 216345.4 & E-Ester-C6-116: Butanoic acid, ethyl ester & 62.88 & 0.488 \\
\hline 14.421 & 19560.1 & E-Ester-C7-130: Pentanoic acid, ethyl ester & 5.09 & 0.545 \\
\hline 20.717 & 280880.3 & E-Ester-C8-142: 2-Hexenoic acid, ethyl ester & 66.56 & 0.599 \\
\hline 15.928 & 71413.4 & E-Ester-C8-142: 3-Hexenoic acid, ethyl ester & 16.92 & 0.599 \\
\hline 18.996 & 346650.8 & E-Ester-C8-142: 3-Hexenoic acid, ethyl ester & 82.15 & 0.599 \\
\hline 16.605 & 384969.1 & E-Ester-C8-144: Pentanoic acid, 3-methyl-, ethyl ester & 92.51 & 0.590 \\
\hline
\end{tabular}




\begin{tabular}{|l|l|l|l|l|}
\hline 18.334 & 395585 & E-Ester-C8-144: Hexanoic acid, ethyl ester & 95.06 & 0.590 \\
\hline 25.608 & 43933 & E-Ester-C10-172: Hexanoic acid, butyl ester & 9.45 & 0.659 \\
\hline 23.394 & 38112.7 & E-Ester-C10-172: Octanoic acid, ethyl ester & 8.20 & 0.659 \\
\hline 25.848 & 45296.7 & E-Ester-C10-172: Octanoic acid, ethyl ester & 9.75 & 0.659 \\
\hline 24.034 & 53467.6 & E-Ester-C13-214: n-Heptyl hexanoate & 10.41 & 0.728 \\
\hline \multicolumn{7}{|l|}{} \\
\hline 3.859 & 210794.6 & E-Ether-C4-74: Ethyl ether & 52.06 & 0.574 \\
\hline 16.237 & 58656.2 & E-Ether-C6-102: Butane, 2-ethoxy- & 11.97 & 0.695 \\
\hline 7.364 & 173169 & E-Ether-C6-118: Ethane, 1,1-diethoxy- & 40.89 & 0.601 \\
\hline \multicolumn{7}{|l|}{} \\
\hline 23.587 & 52873.4 & n-Dodecane (ISTD) & 7.50 & 1 \\
\hline
\end{tabular}




\subsection{Ethanol conversion product list (without lignin)}

Table S2. Ethanol product list obtained from the catalytic reaction of ethanol (without lignin) at $340{ }^{\circ} \mathrm{C}$ for $4 \mathrm{~h}$ over the $\mathrm{Cu}_{20} \mathrm{MgAl}(2)$ catalyst

\begin{tabular}{|c|c|c|c|c|}
\hline $\begin{array}{l}\text { Retention } \\
\text { time (min) }\end{array}$ & Area & Compound Name & $\begin{array}{l}\text { Amount of } \\
\text { products } \\
(\mathrm{mg})\end{array}$ & $\begin{array}{l}\text { Weight RF to } \mathrm{C} 12 \\
\text { Alkane }\end{array}$ \\
\hline 3.34 & 15800.7 & E-Alkane-C2-30: Ethane & 2.25 & 0.946 \\
\hline 3.38 & 23504.1 & E-Alkane-C3-42: Propene & 3.12 & 1.012 \\
\hline 3.48 & 292907.6 & E-Alkene-C4-56:2-Butene, & 38.94 & 1.012 \\
\hline 3.54 & 36067.6 & E-Alkene-C4-56:2-Butene, & 4.79 & 1.013 \\
\hline 3.71 & 179365.8 & E-Alkene-C5-70: 1-Pentene & 23.83 & 1.013 \\
\hline 3.83 & 41306.5 & E-Alkene-C5-70: 2-Methyl-1-butene & 5.49 & 1.013 \\
\hline 3.78 & 100627.7 & E-Alkene-C5-70: 2-Pentene & 13.37 & 1.013 \\
\hline 4.42 & 420831.5 & E- Alkene-C6-84: 2-Hexene & 55.95 & 1.012 \\
\hline 4.56 & 70899 & E-Alkene-C6-84: 2-Hexene & 9.43 & 1.012 \\
\hline 4.63 & 168331.7 & E-Alkene-C6-84: 2-Pentene, 2-methyl- & 22.38 & 1.012 \\
\hline 4.52 & 74995 & E-Alkene-C6-84: 2-Pentene, 3-methyl- & 9.97 & 1.012 \\
\hline 5.68 & 58267.6 & E-Alkene-C7-98: 1-Heptene & 7.75 & 1.011 \\
\hline 5.97 & 52965.8 & E-Alkene-C7-98:2-Heptene & 7.05 & 1.011 \\
\hline 5.91 & 58490 & E-Alkene-C7-98: 2-Heptene & 7.78 & 1.011 \\
\hline 5.78 & 191831.7 & E-Alkene-C7-98: 3-Heptene & 25.52 & 1.011 \\
\hline 12.04 & 66189.4 & E-Alkene-C9-126: 3-Nonene & 8.81 & 1.011 \\
\hline 11.93 & 65139 & E-Alkene-C9-126: 4-Nonene & 8.66 & 1.012 \\
\hline 19.78 & 89978.6 & E-Alkene-C11-154: 5-Undecene & 11.96 & 1.012 \\
\hline 7.77 & 21333568.8 & E-Alcohol-C4-74 : 1-Butanol & 4414.42 & 0.650 \\
\hline 10.05 & 51793.7 & E-Alcohol-C5-88: 1-Butanol, 2-methyl- & 9.83 & 0.709 \\
\hline 8.33 & 222912.7 & E-Alcohol-C5-88: 2-Pentanol & 42.30 & 0.709 \\
\hline 15.18 & 5203217 & E-Alcohol-C6-102 : 1-Hexanol & 933.31 & 0.750 \\
\hline 11.61 & 93686.2 & E-Alcohol-C6-102: 3-Hexanol & 16.80 & 0.750 \\
\hline 13.86 & 1646856.1 & E-Alcohol-C6-102: 1-Butanol, 2-ethyl- & 295.40 & 0.750 \\
\hline 15.32 & 564197.2 & E-Alcohol-C7-116: 4-Heptanol & 97.17 & 0.781 \\
\hline 22.87 & 987294.3 & E-Alcohol-C8-130 : 1-Octanol & 164.62 & 0.807 \\
\hline 21.15 & 490126.1 & E-Alcohol-C8-130: 1-Hexanol, 2-ethyl- & 81.72 & 0.807 \\
\hline 30.01 & 63450.9 & E-Alcohol-C11-172:1-Undecanol & 9.96 & 0.857 \\
\hline 28.28 & 89606.1 & E-Alcohol-C14-214 : 2-Ethyl-1-dodecanol & 13.59 & 0.887 \\
\hline 3.61 & 277875.1 & E-Aldehyde-C2-44-Acetaldehyde & 116.57 & 0.321 \\
\hline 5.25 & 345521.6 & E-Aldehyde-C3-72: Butanal & 117.88 & 0.394 \\
\hline 9.54 & 66277.7 & E-Aldehyde-C6-100 : Butanal, 2-ethyl- & 12.58 & 0.709 \\
\hline 12.45 & 88247.7 & E-Aldehyde-C6-98 : 2-Butenal, 2-ethyl- & 16.41 & 0.723 \\
\hline 17.21 & 59315.7 & E-Aldehyde-C8--128 : Hexanal, 2-ethyl- & 10.31 & 0.774 \\
\hline 24.98 & 8608.1 & E-Aldehyde-C8-128 : Hexanal, 2-ethyl- & 1.50 & 0.774 \\
\hline 5.33 & 4355710 & E-Ester-C4-88: Ethyl acetate & 1816.31 & 0.323 \\
\hline
\end{tabular}




\begin{tabular}{|c|c|c|c|c|}
\hline 10.40 & 1919861.9 & E-Ester-C6-116: Butanoic acid, ethyl ester & 529.24 & 0.488 \\
\hline 15.94 & 97052.2 & E-Ester-C8-142: 3-Hexenoic acid, ethyl ester & 21.81 & 0.599 \\
\hline 19.02 & 2924.5 & E-Ester-C8-142: 3-Hexenoic acid, ethyl ester & 0.66 & 0.599 \\
\hline 19.11 & 819399.7 & E-Ester-C8-144 : Acetic acid, hexyl ester & 186.75 & 0.590 \\
\hline 18.19 & 1308084.9 & E-Ester-C8-144 : Butanoic acid, butyl ester & 298.12 & 0.590 \\
\hline 18.34 & 686896.3 & E-Ester-C8-144: Hexanoic acid, ethyl ester & 156.55 & 0.590 \\
\hline 17.46 & 276966.3 & E-Ester-C8-144 : Acetic acid, 2-ethylbutyl ester & 63.12 & 0.590 \\
\hline 24.25 & 99517.9 & E-Ester-C10-172: Acetic acid, 2-ethylhexyl ester & 20.31 & 0.659 \\
\hline 30.89 & 27899.1 & E-Ester-C10-172 : Acetic acid, 2-ethylhexyl ester & 5.69 & 0.659 \\
\hline 25.44 & 22891.6 & E-Ester-C10-172: Acetic acid, octyl ester & 4.67 & 0.659 \\
\hline 26.58 & 220906.3 & E-Ester-C10-172 : Acetic acid, octyl ester & 45.08 & 0.659 \\
\hline 24.07 & 92565.9 & E-Ester-C10-172 : Butanoic acid, 2-ethyl-, butyl ester & 18.89 & 0.659 \\
\hline 24.72 & 18646.4 & E-Ester-C10-172 : Octanoic acid, ethyl ester & 3.81 & 0.659 \\
\hline 25.60 & 669197.4 & E-Ester-C10-172: Hexanoic acid, butyl ester & 136.56 & 0.659 \\
\hline 25.85 & 195738.9 & E-Ester-C10-172: Octanoic acid, ethyl ester & 39.94 & 0.659 \\
\hline 30.78 & 32522.5 & E-Ester-C12- $200:$ 2-Ethylbutyl hexanoate & 6.18 & 0.708 \\
\hline 30.11 & 92002.6 & E-Ester-C12-200 : Butanoic acid, octyl ester & 17.48 & 0.708 \\
\hline 32.27 & 221787.4 & E-Ester-C12-200 : Butyl caprylate & 42.14 & 0.708 \\
\hline 32.57 & 56779.2 & E-Ester-C12-200 : Decanoic acid, ethyl ester & 10.79 & 0.708 \\
\hline 33.24 & 30844.9 & E-Ester-C14-228 : Lauryl acetate & 4.88 & 0.850 \\
\hline 38.20 & 20448.8 & E-Ester-C14-228 : Octanoic acid, hexyl ester & 3.24 & 0.850 \\
\hline 3.87 & 120624.6 & E-Ether-C4-74: Ethyl ether & 28.25 & 0.574 \\
\hline 6.15 & 81615.9 & E-Ether-C6-102 : Butane, 1-ethoxy- & 15.80 & 0.695 \\
\hline 5.56 & 71532.2 & E-Ketone-C4-72 : 2-Butanone & 16.30 & 0.590 \\
\hline 9.60 & 97892.5 & E-Ketone-C6-100 : 2-Pentanone, 3-methyl- & 18.58 & 0.709 \\
\hline 10.71 & 365158.4 & E-Ketone-C6-100 : 3-Hexanone & 69.30 & 0.709 \\
\hline 12.81 & 63587.3 & E-Ketone-C7-114 : 2-Pentanone, 3-ethyl- & 11.47 & 0.746 \\
\hline 14.06 & 1044682.7 & E-Ketone-C7-114 : 4-Heptanone & 188.49 & 0.746 \\
\hline 19.28 & 111827.8 & E-Ketone-C8-114: 4-Heptanone & 20.18 & 0.746 \\
\hline 16.08 & 205545.7 & E-Ketone-C8-128 : 4-Heptanone, 3-methyl- & 37.09 & 0.746 \\
\hline 18.88 & 85933 & E-Ketone-C8-128: 3-Octanone & 15.50 & 0.746 \\
\hline 23.20 & 79416.3 & E-Ketone-C9-142: 2-Nonanone & 13.38 & 0.799 \\
\hline 22.07 & 613534.3 & E-Ketone-C9-142: 4-Nonanone & 103.36 & 0.799 \\
\hline 26.00 & 53667.7 & E-Ketone-C10-142 : Nonane, 4-methyl- & 9.04 & 0.799 \\
\hline 23.32 & 81822.4 & E-ketone-C10-156 : 3-Isopropyl-5-methylhexan-2-one & 13.47 & 0.817 \\
\hline 23.80 & 61507.5 & E-Ketone-C10-156 : 3-Methyl-4-nonanone & 10.12 & 0.817 \\
\hline 29.33 & 153394 & E-Ketone-C11-170:4-Undecanone & 24.77 & 0.833 \\
\hline 29.26 & 64190 & E-Ketone-C11-170:6-Undecanone & 10.37 & 0.833 \\
\hline 23.59 & 55750.3 & n-Dodecane (ISTD) & 7.50 & 1.000 \\
\hline
\end{tabular}




\subsection{Result of blank reaction (without catalyst)}

Table S3. Results obtained from the blank reaction and catalytic reaction at $340{ }^{\circ} \mathrm{C}$ for $4 \mathrm{~h}$.

\begin{tabular}{|c|c|c|c|c|c|c|}
\hline \multirow{2}{*}{ Entry } & \multirow{2}{*}{ Catalyst } & \multicolumn{5}{|c|}{ Yield lignin products (wt \%) } \\
\hline & & monomers & THF-soluble LR $^{a}$ & THF-insoluble LR $^{b}$ & char & Total yield \\
\hline $1^{\mathrm{a}}$ & Blank & 8 & 15 & 0 & 36 & 59 \\
\hline $2^{a}$ & $\mathrm{Cu}_{20} \mathrm{MgAl}(2)$ & 20 & 69 & 9 & 3 & 101 \\
\hline $3^{\mathrm{b}}$ & $\mathrm{Cu}_{20} \mathrm{MgAl}(2)$ & 30 & 72 & 8 & 0 & 110 \\
\hline
\end{tabular}

${ }^{\mathrm{a}} 50-\mathrm{ml}$ autoclave conditions: $1 \mathrm{~g}$ of lignin, $0.5 \mathrm{~g}$ of catalyst, and $20 \mathrm{ml}$ of solvent. ${ }^{\mathrm{b}} 100-\mathrm{ml}$ autoclave conditions: $1 \mathrm{~g}$ of lignin, 0.5 $\mathrm{g}$ of catalyst, and $40 \mathrm{ml}$ of solvent.

Without catalyst, lignin was only partially depolymerized in ethanol solvent. The main product was char and the monomer yield was only $8 \mathrm{wt} \%$. Among the monomeric products, guaiacol- and syringol-type molecules were predominant and no deoxygenated aromatics or hydrogenated cyclics were formed. The low total yield in blank reaction is probably due to the formation of significant amounts of char, which remained stuck on the internal parts of the batch autoclave reactor system and which could not be recovered completely. ${ }^{1}$ 


\subsection{Identification of peaks in HSQC NMR}
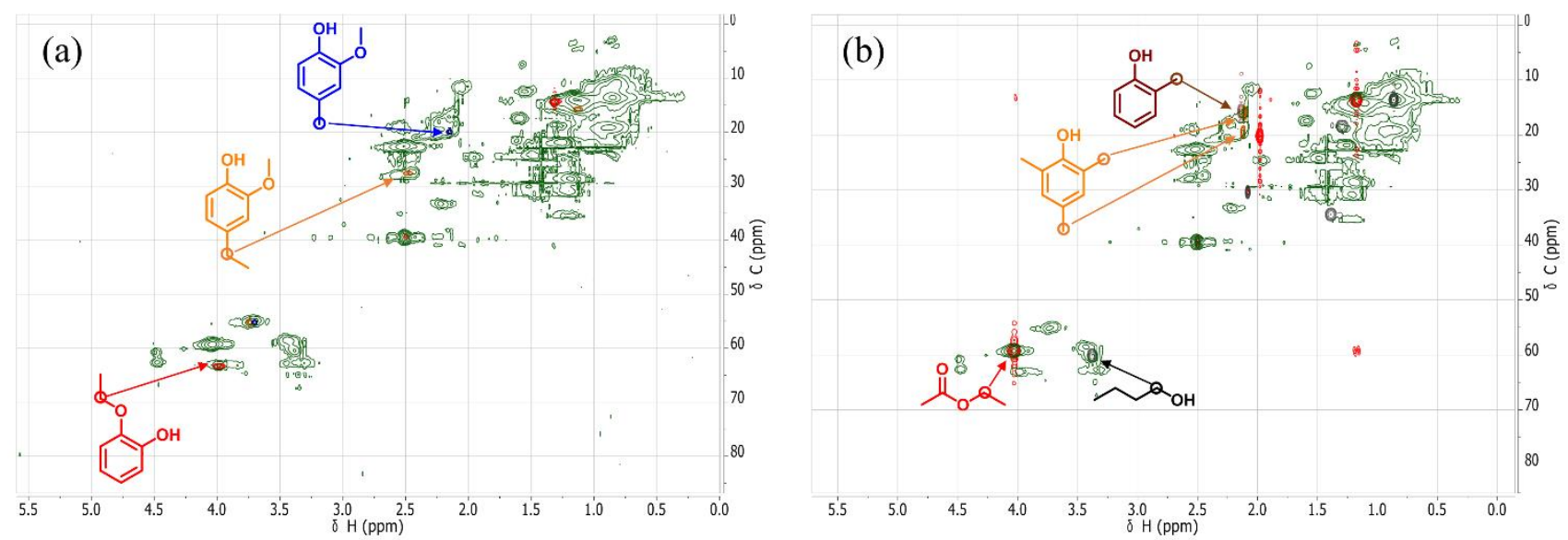

Figure S1. Side-chain region of the of ${ }^{1} \mathrm{H}-{ }^{13} \mathrm{C}$ HSQC NMR spectra of the lignin residue (green colour) following catalytic reaction at $340{ }^{\circ} \mathrm{C}$ for $4 \mathrm{~h}$ over the $\mathrm{CuMgAl}(4)$ catalyst and the standard compounds: (a) 2-ethoxyphenol (red), 4-ethyl guaiacol (orange) and 4-methyl guaiacol (blue); (b) ethyl acetate (red), 1-butanol (black), 2-methyl phenol (brown) and 2,4,6trimethyl phenol (orange).

Standard compounds were used to help to identify the peaks of HSQC NMR spectra. Figure S1 compares the HSQC NMR spectra of lignin residue with some representative standard compounds. 
1.5 XPS analysis of the catalysts.

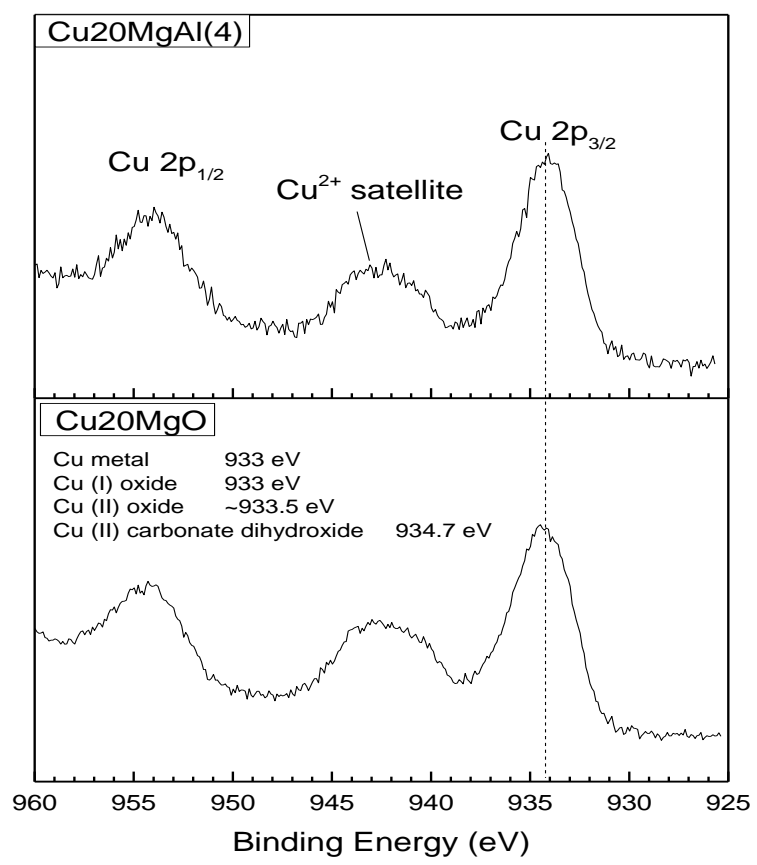

Figure S2. XPS spectra of the $\mathrm{Cu}_{20} \mathrm{MgAl}(4)$ and $\mathrm{Cu}_{20} \mathrm{MgO}$ catalysts.

XPS results show that the binding energy (BE) of $\mathrm{Cu} 2 \mathrm{p}_{3 / 2}$ is around $-934.2 \mathrm{eV}$. Moreover the presence of satellite peak at $943.5 \mathrm{eV}$, suggesting that the $\mathrm{Cu}$ is in the $\mathrm{Cu}^{2+}$ stage. (http://xpssimplified.com/elements/copper.php). 


\subsection{GPC analysis of the lignin and its residues.}

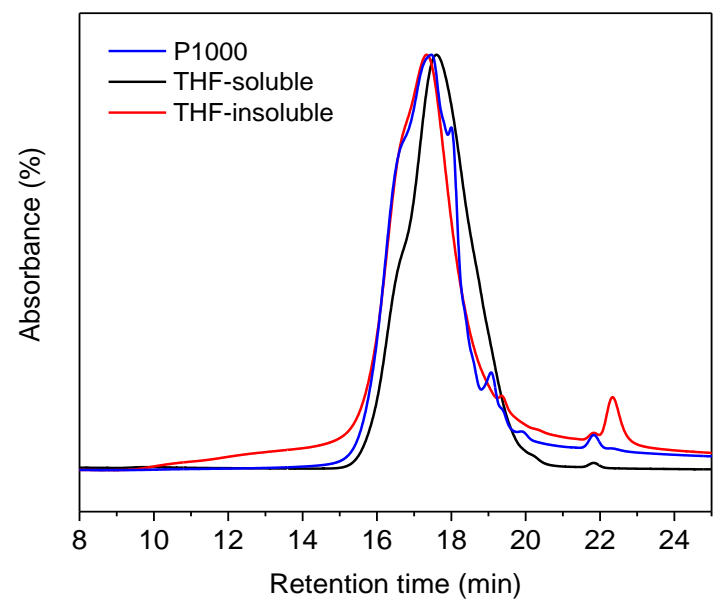

Figure S3. Representative GPC molecular-weight distributions of the THF-soluble fraction of the parent lignin and its lignin residue after reaction in ethanol at $340{ }^{\circ} \mathrm{C}$ for $4 \mathrm{~h}$ over the $\mathrm{Cu}_{20} \mathrm{MgAl}(2)$ catalyst. (Chromatograms have been normalized)

Table S4. GPC analysis of the THF-soluble fraction of the parent lignin and its lignin residue after reaction in ethanol at $340{ }^{\circ} \mathrm{C}$ for 4 h over the $\mathrm{Cu}_{20} \operatorname{MgAl}(2)$ catalyst

\begin{tabular}{|c|c|c|}
\hline Sample & Mn & Mw \\
\hline P1000 & 455 & 1104 \\
\hline THF-soluble residue & 361 & 817 \\
\hline THF-insoluble residue & 449 & 8479 \\
\hline
\end{tabular}

Figure S3 and Table S4 show the representative GPC results of the P1000 lignin and its lignin residues obtained from the case of $\mathrm{Cu}_{20} \operatorname{MgAl}(2)$. The weight average molecular weight is $817 \mathrm{~g} / \mathrm{mol}$, lower than the THF-soluble fraction of P1000 lignin which is $1100 \mathrm{~g} / \mathrm{mol}$. The THF-insoluble residue shows a broad peak at the early elution time region, indicating presence of much heavier products. The Mw of this residue is $8479 \mathrm{~g} / \mathrm{mol}$. We have made an update in the manuscript about this statement. 


\subsection{Gas phase products analysis.}

Table S5. Gas phase products obtained from the reaction of lignin at $340{ }^{\circ} \mathrm{C}$ for $4 \mathrm{~h}$ over the mixed oxide catalysts with different $\mathrm{M}^{2+} / \mathrm{M}^{3+}$ ratios.

\begin{tabular}{|c|c|c|c|c|c|c|c|c|c|c|}
\hline \multirow{2}{*}{ Entry } & \multirow{2}{*}{ Catalyst } & \multicolumn{8}{|c|}{ Amount (mmol) } & \multirow{2}{*}{$\underset{(\mathrm{mmol})}{\text { Sum }}$} \\
\hline & & $\mathrm{CH}_{4}$ & $\mathrm{C}_{2} \mathrm{H}_{6}$ & $\mathrm{C}_{2} \mathrm{H}_{4}$ & $\mathrm{C}_{3} \mathrm{H}_{8}$ & $\mathrm{C}_{3} \mathrm{H}_{6}$ & $\mathrm{CO}_{2}$ & $\mathrm{H}_{2}$ & $\mathrm{CO}$ & \\
\hline 1 & $\mathrm{Cu}_{20} / \gamma-\mathrm{Al}_{2} \mathrm{O}_{3}$ & 1.70 & 3.29 & 5.08 & 0.17 & 0.28 & 2.23 & 7.59 & 1.96 & 22.29 \\
\hline 2 & $\mathrm{Cu}_{20} \operatorname{MgAl}(2)$ & 2.77 & 2.07 & 1.68 & 0.12 & 1.05 & 1.51 & 33.46 & 2.70 & 45.36 \\
\hline 3 & $\mathrm{Cu}_{20} \mathrm{MgAl}(3)$ & 2.34 & 1.79 & 1.54 & 0.12 & 0.87 & 1.86 & 30.76 & 2.50 & 41.79 \\
\hline 4 & $\mathrm{Cu}_{20} \mathrm{MgAl}(4)$ & 2.57 & 1.95 & 1.75 & 0.14 & 0.90 & 1.80 & 34.76 & 2.38 & 46.24 \\
\hline 5 & $\mathrm{Cu}_{20} \mathrm{MgAl}(6)$ & 2.33 & 1.74 & 1.46 & 0.11 & 0.98 & 1.49 & 27.39 & 2.06 & 37.56 \\
\hline 6 & $\mathrm{Cu}_{20} \mathrm{MgO}$ & 2.03 & 1.92 & 2.40 & 0.14 & 2.00 & 1.49 & 22.33 & 1.69 & 34.01 \\
\hline
\end{tabular}

Notes: Reactor volume: $100 \mathrm{ml}$, liquid volume: $40 \mathrm{ml}$, amount of catalyst: $0.5 \mathrm{~g}$.

Table S6. Gas phase products obtained from the reaction of lignin at $340{ }^{\circ} \mathrm{C}$ for $4 \mathrm{~h}$ over the mixed oxide catalysts with different $\mathrm{Cu}$ content.

\begin{tabular}{|c|c|c|c|c|c|c|c|c|c|c|}
\hline \multirow{2}{*}{ Entry } & \multirow{2}{*}{ Catalyst } & \multicolumn{8}{|c|}{ Amount (mmol) } & \multirow{2}{*}{$\underset{(\mathrm{mmol})}{\text { Sum }}$} \\
\hline & & $\mathrm{CH}_{4}$ & $\mathrm{C}_{2} \mathrm{H}_{6}$ & $\mathrm{C}_{2} \mathrm{H}_{4}$ & $\mathrm{C}_{3} \mathrm{H}_{8}$ & $\mathrm{C}_{3} \mathrm{H}_{6}$ & $\mathrm{CO}_{2}$ & $\mathrm{H}_{2}$ & $\mathrm{CO}$ & \\
\hline 1 & $\operatorname{MgAl}(4)$ & 1.00 & 1.16 & 0.83 & 0.09 & 0.22 & 1.56 & 6.81 & 1.19 & 12.85 \\
\hline 2 & $\mathrm{Cu}_{10} \mathrm{MgAl}(4)$ & 2.63 & 2.48 & 2.14 & 0.17 & 1.38 & 1.41 & 24.06 & 2.59 & 36.86 \\
\hline 3 & $\mathrm{Cu}_{20} \mathrm{MgAl}(4)$ & 2.57 & 1.95 & 1.75 & 0.14 & 0.90 & 1.80 & 34.76 & 2.38 & 46.24 \\
\hline 4 & $\mathrm{Cu}_{40} \mathrm{MgAl}(4)$ & 2.63 & 2.45 & 1.61 & 0.16 & 0.95 & 1.88 & 29.97 & 2.73 & 42.38 \\
\hline
\end{tabular}

\section{Reference}

1. Huang, X.; Korányi, T. I.; Boot, M. D.; Hensen, E. J. M. ChemSusChem 2014, 7, 2276-2285. 